\title{
Dynamic system model for ultrasonic lubrication in perpendicular configuration
}

\author{
Sheng Dong, Marcelo J. Dapino* \\ Smart Vehicle Concepts Center, Department of Mechanical and Aerospace Engineering, \\ The Ohio State University, Columbus, OH 43210, USA
}

\begin{abstract}
Ultrasonic lubrication can be achieved by superimposing ultrasonic vibrations onto the relative velocity between two surfaces. Ultrasonic vibrations are typically generated by a piezoelectric actuator. Relative to the macroscopic velocity, the vibrations can be longitudinal, transverse, or perpendicular. Often considered as a purely interfacial effect, ultrasonic lubrication is in fact a system phenomenon incorporating the dynamics of the actuator, sliding surfaces, and surrounding structure. This article presents a dynamic system model for ultrasonic lubrication configured in perpendicular mode, as experimentally measured with a modified pin-on-disc tribometer. The framework includes a lumped-parameter, dynamic model for the tribometer, an electromechanical model for the piezoelectric transducer used to generated the ultrasonic vibrations in the tribometer, and a "cube" model for the contact mechanics between asperities. Electrical impedance, system vibrations, and friction reduction are examined. Results show a strong match between experiments and simulations with errors lower than 10\%. A parametric study is conducted to investigate the influence of driving voltage, macroscopic velocity, driving frequency, and signal waveform on ultrasonic friction reduction.
\end{abstract}

Keywords: System dynamics, ultrasonic lubrication, piezoelectric actuator, friction reduction, parametric study

\footnotetext{
*Corresponding author. Tel: 1 (614) 688-3689

Email address: dapino.1@osu.edu (Marcelo J. Dapino)
} 


\section{1. introduction}

Friction is the resistance to the motion between two contacting surfaces when they slide or roll relative to each other [1]. Ultrasonic lubrication is achieved through superimposition of ultrasonic vibrations onto the macroscopic velocity. Ultrasonic vibrations can be applied in either of three directions: longitudinal, transverse, and perpendicular, as well as combinations thereof. Piezoelectric actuators are often employed to generate the vibrations.

For a given macroscopic velocity, higher vibratory velocity results in greater friction reduction. In a harmonic sense, the magnitude of the vibration velocity is determined by the product of the amplitude and frequency of the vibration displacement. For this reason, compact ultrasonic transducer sources can be utilized to achieve high vibration velocity at small vibration amplitudes. Additionally, ultrasonic frequencies are significantly higher than the natural frequencies of any auxiliary structures. It essentially creates an acoustic isolation to the transducer, which prevents the driving power from leaking to the structures.

Although several factors contribute to ultrasonic lubrication, a reduction in the effective contact pressure between asperities is the dominant factor for friction reduction when the vibrations are perpendicular to the macroscopic velocity. This study is focused on that mode of operation. The degree of friction reduction can be controlled by modulating vibration velocity, which is done by varying the actuator's drive voltage. Variable, solid state lubrication can be used in applications when traditional lubrication is unfeasible (e.g., vehicle seats, space mechanisms) or where friction modulation is desirable (e.g., automotive steering or suspension components).

Both experimental and modeling studies have been conducted to understand and explain ultrasonic lubrication. For example, Littmann et al. [2, 3] used a piezoelectric actuator generating vibrations at $60 \mathrm{kHz}$, making it slide longitudinally on a guide track. They also developed a mathematical relationship between velocity ratio and friction ratio, which indicates that a higher vibration velocity results in a greater friction reduction. In their study, the velocity ratio was defined as the macroscopic velocity over the velocity of the ultrasonic vibrations. The friction ratio was defined as the friction force with ultrasonic vibrations over friction force without ultrasonic vibrations. It was proposed that 
a small velocity ratio leads to a low friction ratio, and hence effective friction reduction. As the velocity ratio increases, so does the friction ratio until a value of 1 is achieved and no further benefit from the ultrasonic vibrations is possible. Therefore, an increase in macroscopic sliding velocity moves the system towards a friction ratio of 1 and reduces the effectiveness of the ultrasonic vibrations.

Kumar and Hutchings [4] studied the mechanisms of friction reduction with ultrasonic vibrations applied longitudinal and transverse relative to the macroscopic velocity, respectively. Coulomb friction [5] was employed in their study, which assumes a constant coefficient of friction during sliding. The superposition of ultrasonic vibrations both in longitudinal and transverse directions changes the direction of instantaneous velocity so that the overall magnitude of the friction force is reduced. A similar explanation was proposed by Popov et al. [6] and Tsai et al. [7].

Bharadwaj and Dapino [8, 9, 10] and Gutowski and Leus [11] adopted Dahl's [12] friction model and built it into their dynamic models. Bharadwaj and Dapino $[8,9,10]$ analyzed the influence of contact stiffness, global stiffness, mass, coefficient of friction, and signal waveform on friction reduction. Gutowski and Leus [11] simulated timedependent friction as the output of the dynamic system, and obtained good agreement between the simulation and experimental data. However, in both studies, they treated contact stiffness as a constant value as opposed to a changing parameter when ultrasonic vibrations are present. They provided no physical explanation for the calculation of the contact stiffness, but presented it as a manipulated value for matching the experimental data. Nonetheless, these models were successful in explaining ultrasonic friction reduction with vibrations applied longitudinal to the sliding direction.

Teidelt et al. [13] extended Popov's modeling work from in-plane to out-of-plane. The pin-on-disc set-up was adopted, and ultrasonic vibrations were applied on the pin in a direction perpendicular to the disc surface. They employed the Coulomb friction model, and explained the friction reduction as a result of reduced normal load. The change of the normal load was calculated as the product of the contact stiffness and the deformation in the vertical direction. Dong and Dapino $[14,15,16,17]$ have reported experimental work on ultrasonic friction reduction in the perpendicular direction due to longitudinal vibrations, i.e., through the Poisson effect [14]. They also studied ultrasonic wear reduction 
at different speeds, which was conducted between stainless steel and aluminum using a modified pin-on-disc tribometer $[15,16]$. In addition to experimental work, an elasticplastic "cube" model was proposed to explain the mechanisms of ultrasonic friction and wear reduction when ultrasonic vibrations are employed in the direction vertical to the macroscopic velocity [17].

Prior work on ultrasonic lubrication has shown compelling evidence that the degree of friction reduction is intrinsically dependent on the ratio of the macroscopic sliding speed and the wave propagation speed. This dependency is at the core of why ultrasonic (as opposed to subsonic) vibrations are required in the first place, and puts the speed ratio front and center of any discussion dealing with ultrasonic lubrication. Much of the literature $[2,3,4,6,14,17]$ on this topic considers pseudo-static (i.e., steady state) responses and ignores the dynamics of the system and how the system affects ultrasonic lubrication. This is where the need for dynamic modeling comes in because the sliding and ultrasonic velocities are system-dependent. The focus of this paper is the development of a dynamic, system level model for a modified pin-on-disc tribometer. The approach presented here can be adapted to describe constructive details of other ultrasonic lubrication systems.

In order to explain ultrasonic lubrication at the system level, models must represent these components at three representative scales: a "cube" model for the asperities located at the interface incorporating contact and friction reduction mechanisms, an electromechanical model for the piezoelectric transducer, and a dynamic system model for the overall system. It is apparent that constructive details determine the dynamic response at each of the three scales. In lieu of a generic structure, the model presented here aims to represent the dynamics of a pin-on-disc tribometer system that is the subject of the experiments used to guide and validate the model. Generalization to other structures can follow from the relatively simple construct presented in this study. The model is described in Section 2, followed in Section 3 by a description of experiments conducted on a pin-on-disc tribometer. Model results and comparisons with experimental measurements are provided in Section 4. In Section 5, the model is used to investigate the dependence of ultrasonic lubrication on key system parameters: driving voltage, velocity, driving frequency, and signal waveform. 


\section{Model Development}

\subsection{System dynamics model}

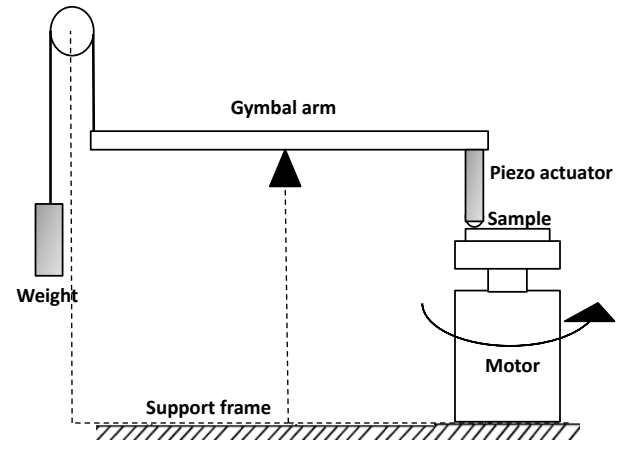

(a)

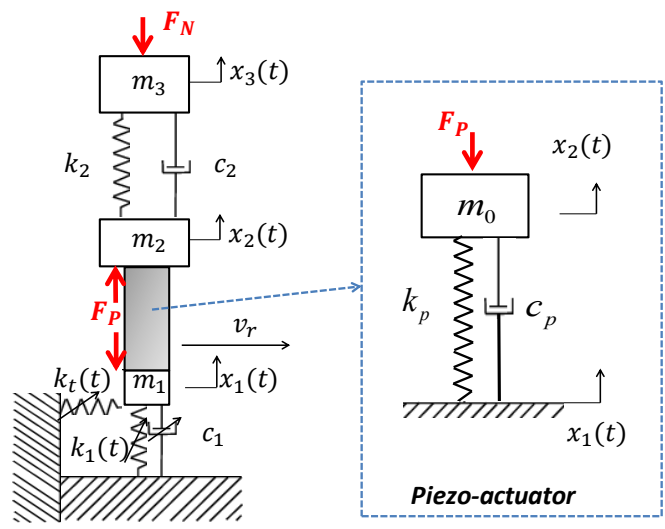

(b)

Figure 1: Model development: (a) schematic of the modified pin-on-disc tribometer utilized in this study; (b) corresponding system dynamics model.

As shown schematically in Fig. 1 (a), a stationary pin is in contact with a rotating disc sample. The pin consists of a piezoelectric actuator and an acorn nut tip. The top end of the pin is fixed to a gymbal arm which is connected to a weight through a pulley system, which is employed to apply normal load to the contact interface.

A lumped-parameter, three-degree-of-freedom system model of the tribometer is shown in Fig. 1 (b). The model consists of a piezoelectric transducer, three masses, springs, and dampers. Mass $m_{1}$ represents the acorn nut connected to the rotating sample (ground) via an axial stiffness $\left(k_{1}\right)$, an axial damping coefficient $\left(c_{1}\right)$, and a tangential stiffness $\left(k_{t}\right)$. The relative velocity between the two surfaces is denoted $v_{r}$ and the normal force at the interface is $F_{N}$. The effective dynamic mass, stiffness, and damping of the weight and pulley system are $m_{3}, k_{2}$ and $c_{2}$, respectively. The equivalent parameters for the piezo-actuator are $m_{0}, k_{p}$, and $c_{p}$. The actuation force generated by the actuator is $F_{P}$, acting on $m_{1}$ and $m_{2}$. The effective dynamic mass of the gymbal arm is represented by $m_{2}$. The displacement of the acorn nut, the gymbal arm, and the weight are $x_{1}, x_{2}$, and $x_{3}$, respectively. 
Equations of motion of the system can be written as

$$
\begin{gathered}
{\left[\begin{array}{ccc}
m_{1} & 0 & 0 \\
0 & m_{2}+m_{0} & 0 \\
0 & 0 & m_{3}
\end{array}\right]\left[\begin{array}{c}
\ddot{x_{1}} \\
\ddot{x_{2}} \\
\ddot{x_{3}}
\end{array}\right]+\left[\begin{array}{ccc}
c_{1}+c_{p} & -c_{p} & 0 \\
-c_{p} & c_{p}+c_{2} & -c_{2} \\
0 & -c_{2} & c_{2}
\end{array}\right]\left[\begin{array}{c}
\dot{x_{1}} \\
\dot{x_{2}} \\
\dot{x_{3}}
\end{array}\right]} \\
+ \\
{\left[\begin{array}{ccc}
k_{1}+k_{p} & -k_{p} & 0 \\
-k_{p} & k_{p}+k_{2} & -k_{2} \\
0 & -k_{2} & k_{2}
\end{array}\right]\left[\begin{array}{l}
x_{1} \\
x_{2} \\
x_{3}
\end{array}\right]=\left[\begin{array}{c}
-F_{P} \\
F_{P} \\
-F_{N}
\end{array}\right],}
\end{gathered}
$$

\subsection{Piezoelectric transducer}

Piezoelectric materials transform energy between electrical and mechanical domains. Although piezoelectric materials are intrinsically nonlinear, transducers based on these materials are often driven at low signal regimes under mechanical bias conditions, leading to approximately linear responses. This situation has been modeled extensively [18], as follows:

$$
\mathbf{D}=\epsilon^{\mathbf{T}} \mathbf{E}+\mathbf{d} \mathbf{T}
$$

and

$$
\mathbf{S}=\mathbf{d E}+\mathbf{s}^{\mathbf{E}} \mathbf{T}
$$

where $\mathbf{D}$ is electric displacement, $\mathbf{T}$ is stress, $\mathbf{E}$ is electric field, $\mathbf{S}$ is strain, $\epsilon^{\mathbf{T}}$ is permittivity at constant stress, $\mathbf{s}^{\mathbf{E}}$ is mechanical compliance at constant electric field, and $\mathbf{d}$ is a coupling constant. In relation to model illustrated in Fig. 1 (b), only the quantities in the poling direction (33) are taken into consideration.

Electromechanical coupling is shown in Fig. 2, where $F$ is mechanical load, $v$ is velocity, $Z_{m}$ is blocked mechanical impedance, $V$ is driving voltage, $I$ is current, $Z_{e}$ is blocked electrical impedance, and $T_{m e}$ and $T_{e m}$ are electromechanical transduction coefficients. Following the procedure of (for instance) Downey and Dapino [19], the coupling equations between electrical and mechanical are

$$
V=Z_{e} I+T_{e m} v
$$

and

$$
F=T_{m e} I+Z_{m} v
$$




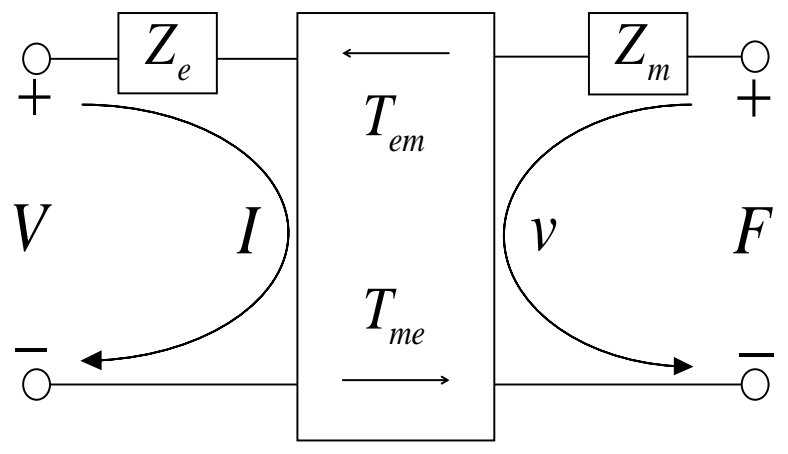

Figure 2: Electromechanical coupling of piezoelectric transducers [18].

where $Z_{e}$ is calculated as

$$
Z_{e}(s)=\frac{1}{C s}
$$

where $C$ is the blocked capacitance of the stack; mechanical capacitance $Z_{m}$ is calculated as

$$
Z_{m}(s)=m_{0} s+c_{p}+k_{p} / s
$$

$T_{e m}$ and $T_{e m}$ are calculated as

$$
T_{e m}(s)=T_{e m}(s)=\frac{d_{33}}{s N \epsilon^{S} S^{E}},
$$

where $\epsilon^{S}=\epsilon^{T}\left(1-k^{2}\right)$, and $k$ is the electromechanical coupling factor of the piezoceramic.

It is assumed that force $F$ acts on impedance $Z_{L}$. The equivalent circuit of the dynamic system is shown in Fig. 3. Due to the nonlinearity of the spring $k_{1}$ and damper $c_{1}$, they are not considered in the equivalent circuit. Therefore, the system is reduced to represent the piezo-actuator with one end connected to the structure and the other end free. The load impedance can be expressed as

$$
Z_{L}(s)=\left[\frac{1}{m_{1} s}+\frac{1}{m_{2} s+Z_{0}}\right]^{-1}
$$

where

$$
Z_{0}=\left[\frac{1}{m_{3} s+F_{N} / s}+\frac{1}{c_{2}+k_{2} / s}\right]^{-1} .
$$

Therefore, the total electrical impedance of the actuator is

$$
Z_{e e}(s)=Z_{e}+\frac{-T_{m e} T_{e m}}{Z_{m}+Z_{L}},
$$




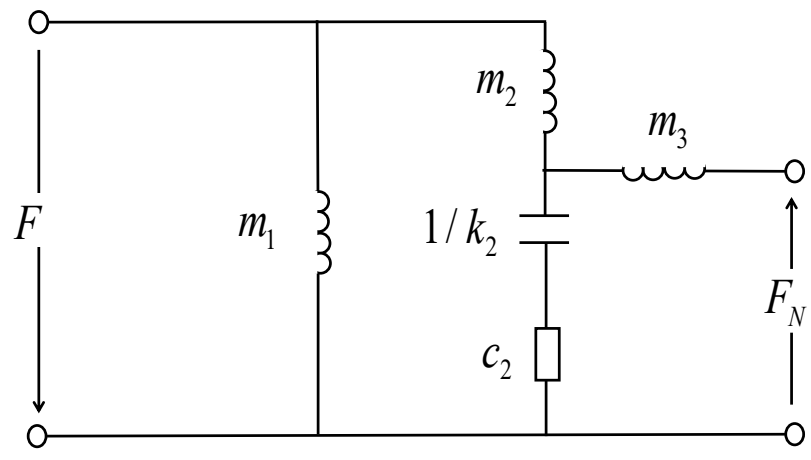

Figure 3: Electrical equivalent circuit of the mechanical system connected to the piezo-actuator.

with the actuation force generated by the actuator given by

$$
F=N d k_{p} V
$$

where $N$ is the number of the wafers in the stack.

\subsection{Elastic-plastic "Cube" model}

The actual contact between two nominally flat surfaces takes place on the surface asperities, as shown in Fig. 4 (a). An elastic-plastic "cube" model was proposed by Dong and Dapino [15] to represent all the contacting asperities. The top area of the deformed cube, $A_{r}(t)$, is the actual contact area, and the height of the cube, $d(t)$, is the distance between the two surfaces. The top area $A_{r}(t)$ is calculated as

$$
A_{r}(t)=A_{p}(t)+A_{e}(t)
$$

where $A_{p}(t)$ is the area associated with plastic deformation of the asperities and $A_{e}(t)$ is the area associated with their elastic deformation. As the distance between the two contacting surfaces changes, $A_{p}(t)$ and $A_{e}(t)$ change as well. Detailed calculations for $A_{e}(t), A_{p}(t)$, and $d(t)$ were presented by Shi and Polycarpou [20] as an enhanced version of the classic Greenwood-Williamson model. It is emphasized that both plastically and elastically deformed asperities contribute to the total actual contact area. 
Deforming asperities provide stiffness on both axial and tangential directions, which are expressed as $k_{1}(t)$ and $k_{t}(t)$, respectively, in the system dynamics model (Fig. 1 (b)). Based on the geometry of the cube, axial stiffness, $k_{1}(t)$, is calculated as

$$
k_{1}(t)=\frac{E^{*} A_{r}(t)}{d(t)},
$$

where $E^{*}$ is the combined Young's modulus of the two materials that are in contact. When the distance between two surfaces changes due to ultrasonic vibrations, the height and the top area of the cube change accordingly but nonlinearly, which results in nonlinearity in $k_{1}(t)$. When the cube height is smaller, the top area increases at a higher rate, thus the stiffness increases. Tangential stiffness $\left(k_{t}(t)\right)$, which is also nonlinear, is calculated as

$$
k_{t}(t)=\frac{E^{*} A_{r}(t)^{2}}{d(t)^{3}}
$$

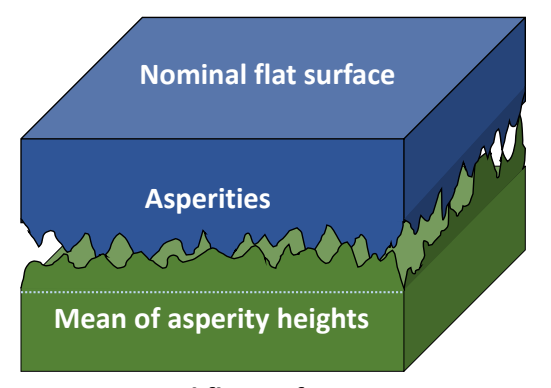

Nominal flat surface

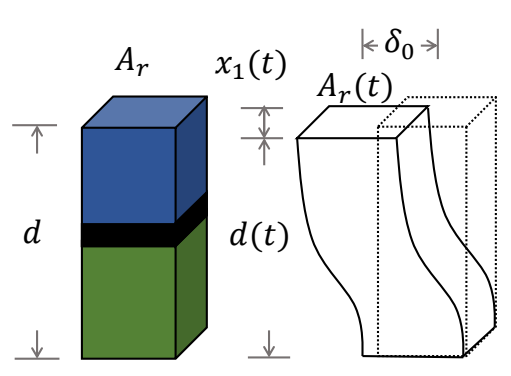

(b) (c)

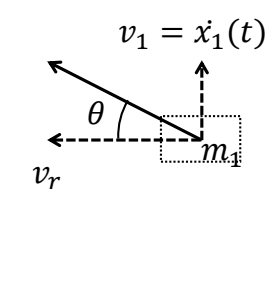

(d)

Figure 4: "Cube" model: (a) asperities between two surfaces; (b) undeformed "cube"; (c) "cube" under elastic-plastic deformation ( $\delta_{0}$ denotes the intrinsic deformation with no ultrasonics applied to the cube); (d) velocity vector at the interface: $v_{r}$ is the macroscopic relative velocity and $v_{1}$ is the ultrasonic vibratory velocity.

Under normal load $F_{N}$, axial and tangential stiffness at equilibrium $\left(x_{1}=0\right)$ are 
denoted as $k_{10}$ and $k_{t 0}$, which are calculated from a cube with top area $A_{r 0}$ and height $d_{0}$ (Fig. 4 (b)). Friction comes from the resistance created by the deformed contacting asperities in the direction opposite to the relative velocity. Intrinsic friction $\left(F_{t 0}\right)$, tangential deformation $\left(\delta_{0}\right)$, and tangential stiffness $\left(k_{t 0}\right)$ are related by

$$
F_{t 0}=k_{t 0} \delta_{0}
$$

It is assumed that tangential deformation of the asperities $\left(\delta_{0}\right)$ is only dependent on the macroscopic velocity $\left(v_{r}\right)$ and remains constant despite the application of ultrasonic vibrations. When ultrasonic vibrations are applied, relative displacement between two surfaces in the vertical direction $\left(x_{1}(t)\right)$ changes the geometry of the cube, hence, the tangential and axial stiffnesses change as well. When two surfaces move away from each other $\left(x_{1}>0\right)$, more asperities stop being in contact, which results in a taller cube with smaller top area. On the other hand, when two surfaces move towards each other $\left(x_{1}<0\right)$, more asperities come into contact, which results in a shorter cube with larger top area.

Instantaneous velocity is at an angle with the tangential direction $(\theta(t))$ once ultrasonic vibrations are applied (Fig. 4 (d)),

$$
\theta(t)=\cos ^{-1}\left[\frac{v_{r}}{v_{1}(t)}\right] .
$$

Therefore, the instantaneous friction force is the projection of the resisting force in the tangential direction,

$$
F_{t}(t)=k_{t}(t) \delta_{0} \cos [\theta(t)]
$$

The overall friction force is the average of the instantaneous friction force over the vibration period $T$,

$$
F_{t}=\frac{1}{T} \int_{0}^{T} F_{t}(t) d t
$$

\section{Experiment}

A modified pin-on-disc tribometer, shown in Fig. 5, was employed to study friction and wear reduction using ultrasonic lubrication. The pin consists of a compressed piezoelectric actuator and an acorn nut. The pin is held by a gymbal assembly, which is also 
used for applying various normal loads and measuring friction forces. The total electrical impedance of the actuator was measured with the acorn nut end vibrating free. The actuator generates vibrations with amplitude of $2.5 \mu \mathrm{m}$ at a frequency of $22 \mathrm{kHz}$. The acorn nut and the discs are made of stainless steel and aluminum, respectively. The total normal force at the interface is $3 \mathrm{~N}$, which includes the a $2 \mathrm{~N}$ weight added to the gymbal in addition to the weight of the piezo-actuator and the gymbal arm.

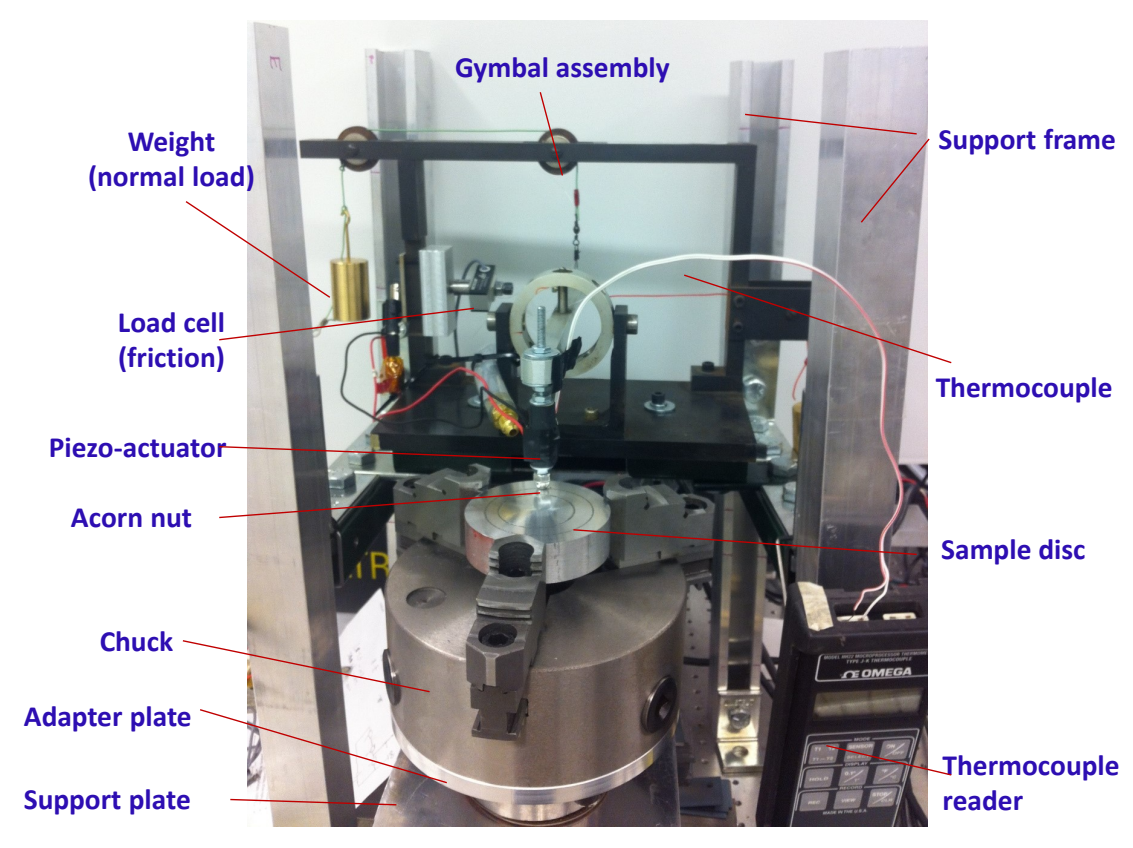

Figure 5: Experimental set-up.

To study the dependence of friction and wear reduction on linear velocity, three different speeds $(20.3,40.6$, and $87 \mathrm{~mm} / \mathrm{s})$ were applied to the disc by changing the rotational speed of the motor. For all three groups, the number of disc revolutions and the travel distance of the pin were chosen as 1600 and $292.5 \mathrm{~m}$, respectively. The nominal rotation diameter was $50 \mathrm{~mm}$. Other parameters such as normal force, vibration frequency, and vibration amplitude remained the same for all tests.

The friction force was sampled at a frequency of $400 \mathrm{~Hz}$ utilizing 2-second sampling 


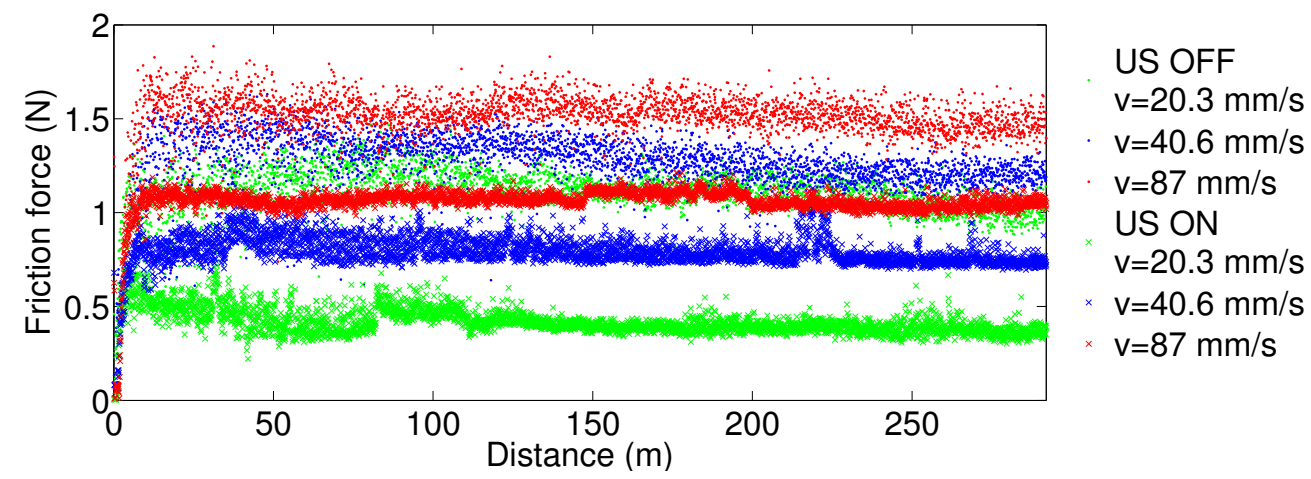

Figure 6: Friction measurements.

windows. The mean values of friction force were calculated and plotted against pin travel distance in Fig. 6. The intrinsic friction forces are represented by dots in the figure, while friction forces with ultrasonic vibrations are shown with " $\mathrm{x}$ " markers. Green, blue and red are used respectively for the test data at $20.3 \mathrm{~mm} / \mathrm{s}, 40.6 \mathrm{~mm} / \mathrm{s}$, and $87 \mathrm{~mm} / \mathrm{s}$. The friction force from each test remains virtually constant once steady-state operation is achieved.

Disc run-out causes inertial acceleration from vertical motion of the pin and gymbal assembly, which results in variation of the normal force. As a result, the measured friction force fluctuates. Figure 6 shows that the friction force is higher at higher linear speeds, whether or not ultrasonic vibrations are applied to the system.

The friction reduction percentage is defined as

$$
P_{F}=\frac{F_{t 0}-F_{t}}{F_{t 0}} \times 100 \%,
$$

where $F_{t 0}$ is the intrinsic friction force and $F_{t}$ is the friction force with ultrasonic vibrations. Ultrasonic vibrations reduce the steady-state friction force for all three linear speeds, however, the percentage decreases as the linear velocity increases.

\section{Computational results}

Total electrical impedance of the piezo-actuator was measured. The measurement was employed to identify of the critical parameters of the model, by matching the computational curve with the measured curve (Fig. 7). These parameters, along with others used 
in the simulation, are listed in Table 1. The simulation was conducted using Simulink in MATLAB.

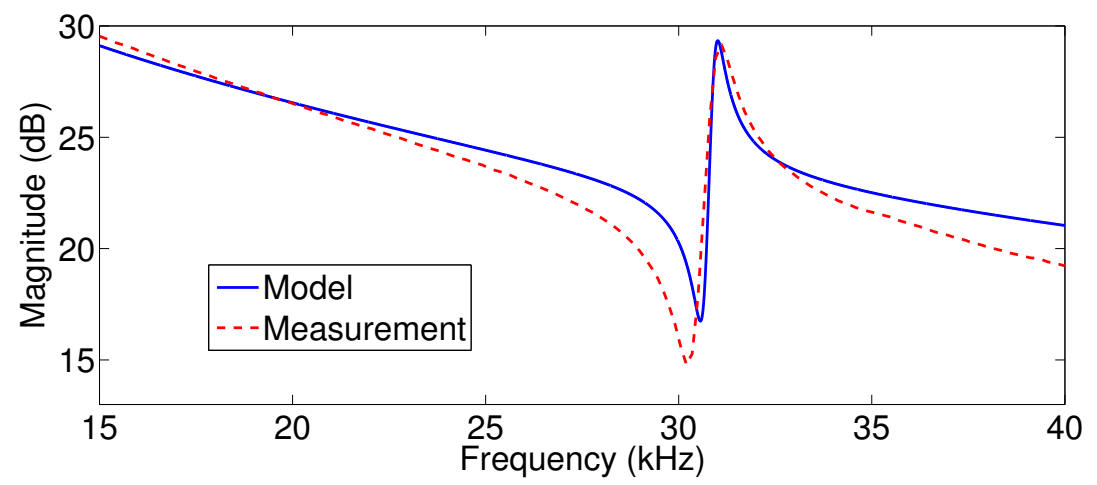

Figure 7: Electrical impedance of the piezoelectric actuator.

Table 1: Parameters used in the simulation.

\begin{tabular}{|c|c|c|}
\hline Pable 1: Parameters used in the simulation. \\
\begin{tabular}{|c|c|c|}
\hline Parameter & Symbol & Value \\
\hline \hline Deviation of asperities heights & $R_{q}$ & $6 \mu \mathrm{m}$ \\
\hline Capacitance of the piezoelectric stack & $C$ & $360 \mathrm{nF}$ \\
\hline Electromechanical coupling factor & $k$ & 0.431 \\
\hline Piezoelectric constant & $d$ & $140 \times 10^{-12} \mathrm{mV}$ \\
\hline Number of layers & $N$ & 62 \\
\hline Equivalent stiffness of the piezo-actuator & $k_{p}$ & $2 \times 10^{9} \mathrm{~N} / \mathrm{m}$ \\
\hline Equivalent mass of the piezo-actuator & $m_{0}$ & $0.07 \mathrm{~kg}$ \\
\hline Equivalent damping coefficient & $c_{p}$ & 30 \\
\hline
\end{tabular}
\end{tabular}

The simulation results of the system vibrations are shown in Fig. 8. Plot (a) shows the overall displacement at $x_{1}$ and $x_{2}$. It takes approximately 0.05 seconds for the vibrations to reach steady state. Steady-state vibrations consist of vibrations at drive frequency and fluctuations at approximately $100 \mathrm{~Hz}$ (plot (b)). Vibrational amplitude at steady-state $\left(x_{1}\right)$ is approximately $2.5 \mu \mathrm{m}$, which is equal to the value measured by a laser vibrometer.

A comparison between the experimental data and the model prediction regarding 


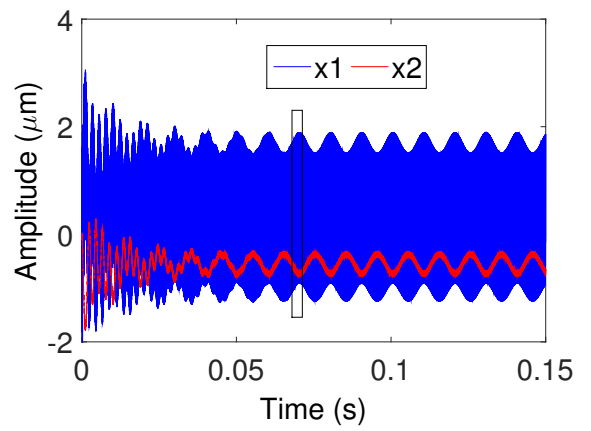

(a)

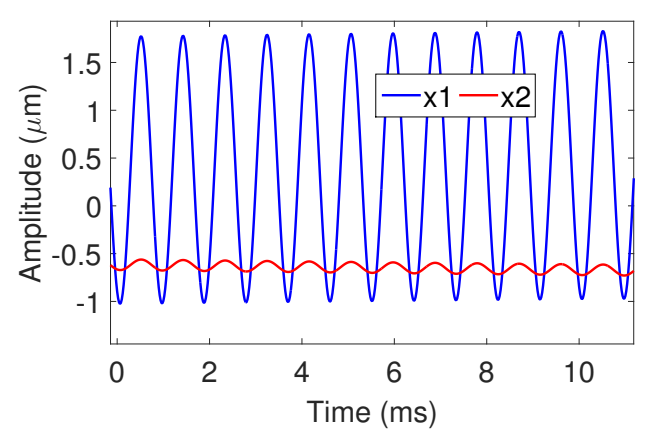

(b)

Figure 8: Simulation results of system vibrations: (a) displacement; (b) zoomed-in displacement.

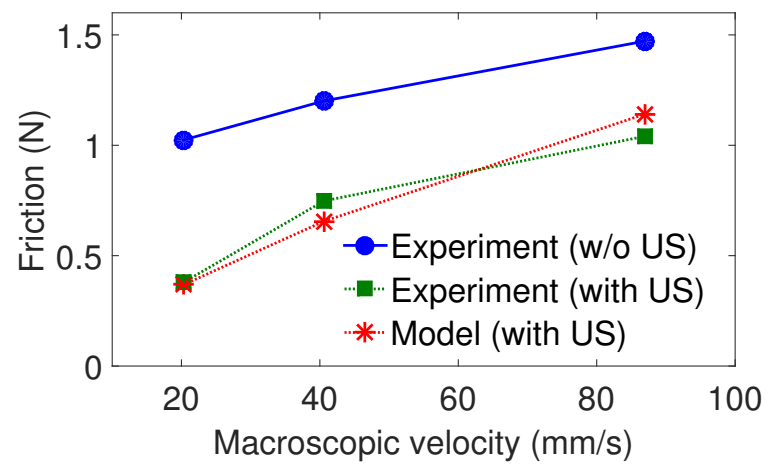

Figure 9: Comparison between friction from measurements and simulation.

reduction of friction is conducted to validate the model. The friction forces measured at three velocities, with and without ultrasonic vibrations, are plotted as dots and squares in Fig. 9. The reduced friction forces calculated from the model are plotted with stars. The comparison shows that the simulation matches the experimental data well at all three velocities, with errors lower than $10 \%$.

\section{Parametric study}

Parameters such as driving voltage, macroscopic velocity, driving frequency, and signal waveform are studied. Friction ratio is defined to indicate the effectiveness of the 
friction reduction, as

$$
\gamma=F_{t} / F_{t 0} .
$$

Lower friction ratio suggests better friction reduction.

Driving voltage

Simulations are conducted at different levels of voltages, from 0 to $200 \mathrm{~V}$ peak-topeak. The displacement $x_{1}$, velocity $v_{1}$, tangential stiffness, and instantaneous friction in one period of ultrasonic vibration are shown in Fig. 10 (a)-(d).

Plots show that both the steady-state displacement and velocity at the interface are sinusoidal. Higher driving voltage results in higher amplitudes of displacement and velocity. When two surfaces move towards each other $\left(x_{1}<0\right)$, a decrease in the cube height, an increase in the top area, and an increase in tangential stiffness occur. The tangential stiffness and instantaneous friction force reach their peaks when $x_{1}$ is at its minimum. The contact between two surfaces is reduced when they move away from each other $\left(x_{1}>0\right)$. Once the dynamic load introduced by the ultrasonic vibrations overcomes the static normal load, the surfaces separate and hopping takes place. This occurs when $x_{1}$ is greater than $3.8 \mu \mathrm{m}$ (dashed line in Fig. 10 (a)) and $k_{t}$ becomes virtually zero (Fig. $10(\mathrm{c})$ ).

The relationship between friction ratio and driving voltage is plotted in Fig. 10 (e). The friction force drops significantly when driving voltage increases from 0 to $20 \mathrm{~V}$, but the friction reduction becomes less significant when the voltage continues to increase over $20 \mathrm{~V}$. It is concluded that higher driving voltage results in more friction reduction. However, voltage increases become less effective in achieving friction reduction.

\section{Macroscopic velocity}

Macroscopic velocity is critical to ultrasonic friction reduction. There have been studies on the relationship between friction reduction and velocity when ultrasonic vibrations were applied in the direction longitudinal to the macroscopic velocity. It was proposed that ultrasonic vibrations can change the direction of the friction force, hence, the overall magnitude of friction. Higher macroscopic velocity results in less friction reduction [2].

In this study, ultrasonic vibrations are out-of-plane perpendicular to the macroscopic velocity. Different voltages are employed for the simulation, from $0 \mathrm{~V}$ to $200 \mathrm{~V}$ peak- 
to-peak. Different voltages result in a different vibratory velocity. A velocity ratio is defined, as

$$
\zeta=v_{r} / v_{1}
$$

where $v_{r}$ is the macroscopic velocity (chosen to be $0 \mathrm{~m} / \mathrm{s}$ to $1 \mathrm{~m} / \mathrm{s}$ ), and $v_{1}$ is the vibrational velocity obtained from the simulation. The relationship between the friction ratio and the velocity ratio are plotted in Fig. 11.

For all voltages, the trend is that higher macroscopic velocity leads to lower friction reduction, which is in line with the experimental data and previous studies of ultrasonic vibrations in a longitudinal direction. For a certain macroscopic velocity, a higher driving voltage results in a higher friction reduction.

\section{Driving frequency}

The driving frequency of the actuator is generally set to be at the resonant frequency of the system in order to maximize the vibration. The relationships between friction ratio and driving frequency $(1-100 \mathrm{kHz})$ at different voltages are plotted in Fig. 12 (a).

It is evident that, for all voltages, the relationship between friction ratio and frequency has a "v" shape: the maximum friction reduction takes place at the resonance. Higher amplitude results in higher maximum reduction. One observation from this plot is that, to achieve same level of friction reduction, the actuator can be driven either at a lower frequency with a higher amplitude, or at a higher frequency with a lower amplitude. There is no singular solution. However, if the power consumption is taken into consideration, an optimum does exist. Power consumption of the actuator is calculated as

$$
P=C U_{p p} U_{\max } f
$$

where $C$ is the capacitance of the actuator, $U_{p p}$ and $U_{\max }$ are the peak-to-peak and maximum voltage applied to the actuator, respectively, and $f$ is the driving frequency.

The relationships between the friction ratio and average power at different voltages are plotted in Fig. 12 (b). For each voltage, the friction ratio curve remains a "v" shape. The tips of the " $\mathrm{v}$ "s form an envelope curve. It indicates that, to achieve a certain level of friction reduction at a certain given voltage, the solution with the least power 
consumption is to drive it at the resonance frequency. An equal amount of reduction may be achieved by driving the actuator at a higher voltage and a non-resonant frequency, but more power is then consumed.

\section{Signal waveform}

In the experiment, sinusoidal waveform is adopted for driving the piezo-actuator. Here, four types of waveforms are compared: sawtooth, square, triangle, and sinusoid. As shown in Fig. 13 (a), the amplitude and the frequency of the signals remain equal: 0-200 V peak-to-peak and $22 \mathrm{kHz}$. The macroscopic velocity is $20.3 \mathrm{~mm} / \mathrm{s}$.

For all four waveforms, the steady-state vibrations in one period are plotted in Fig. 13 (b). It can be seen that all vibrations follow sinusoidal patterns, despite the different waveforms of the driving signals. A square waveform generates the highest vibrational amplitude at the interface, and therefore, leads to highest amount of friction reduction (Fig. 13 (c)). The remaining waveforms that give the highest to lowest reduction are sinusoid, triangle, and sawtooth.

\section{Concluding Remarks}

A dynamic model was proposed in a general form to represent ultrasonic lubrication systems. The model consists of components at different scale levels. A system dynamics model is employed to represent the lubrication system. An electromechanical model is utilized for the piezoelectric actuator. The contact between two surfaces, of which the friction is aimed to be reduced, is modeled by the "cube" model.

An electrical impedance measurement was taken to identify critical model parameters. The simulation results were validated by the experimental data taken from the modified tribometer tests. The simulations match well with the measurements, with all errors lower than $10 \%$.

The influence of driving voltage, macroscopic velocity, driving frequency, and signal waveform on friction reduction is studied. Higher driving voltage results in greater friction reduction. Higher macroscopic velocity leads to a lower reduction of friction. Driving the actuator at its resonant frequency is the most effective solution and requires the least amount of power. When driving at the same peak-to-peak voltages, all waveforms result 
in friction reduction, and can be ranked in the following order from highest to lowest: square, sinusoid, triangle, and sawtooth.

\section{ACKNOWLEDGEMENTS}

The authors would like to acknowledge Tim Krantz from NASA Glenn Research Center, Ryan Hahnlen and Duane Detwiler from Honda R\&D Americas, Inc for their technical support and in-kind contributions. Financial support for this research was provided by the member organizations of the Smart Vehicle Concepts Center (www.SmartVehicleCenter.org), a National Science Foundation Industry/University Cooperative Research Center (I/UCRC). S.D. was supported by a Smart Vehicle Concepts Graduate Fellowship and a Presidential Fellowship from the Ohio State University.

\section{References}

[1] Bhushan, B., "Introduction to tribology," John Wiley \& Sons, New York (2002).

[2] Littmann, W., Storck, H., and Wallaschek, J., "Sliding friction in the presence of ultrasonic oscillations: superposition of longitudinal oscillations," Arch. Appl. Mech. 71, 549-554 (2001).

[3] Storck, H., Littmann, W., Wallaschek, J., and Mracek, M., "The effect of friction reduction in presence of ultrasonic vibrations and its relevance to travelling wave ultrasonic motors, "Ultrasonics, 40, 379-383 (2002).

[4] Kumar, V. and Hutchings, I., "Reduction of the sliding friction of metals by the application of longitudinal or transverse ultrasonic vibration," Tribol. Int., 37, 833-840 (2004).

[5] Coulomb, C., "The theory of simple machines (in French)," Memories of Math and Physics Academy of Science, 10, 161-331 (1785).

[6] Popov, V., Starcevic, J., and Filippov, A., "Influence of Ultrasonic In-Plane Oscillations on Static and Sliding Friction and Intrinsic Length Scale of Dry Friction Processes, " Tribol. Lett., 39, 25-30 (2010).

[7] Tsai, C. and Tseng C. "The effect of friction reduction in the presence of in-plane vibrations," Archive of Applied Mechanics, 75, 164-176 (2005).

[8] Bharadwaj, S. and Dapino, M., "Effect of load on active friction control using ultrasonic vibrations," Proc. SPIE, 7290, 72900G (2009).

[9] Bharadwaj, S. and Dapino, M., "Friction control in automotive seat belt systems by piezoelectrically generated ultrasonic vibrations," Proc. SPIE, 7645, 76450E (2010).

[10] Bharadwaj, S. and Dapino, M., "Characterization of friction reduction with tangential ultrasonic vibrations using a SDOF model," International Journal of Vehicle Design, 63 (2/3) (2013). 
[11] Gutowski, P. and Leus, M., "The effect of longitudinal tangential vibrations on friction and driving forces in sliding motion," Tribology International, 55, 108-118 (2012).

[12] Dahl, P., " A solid friction model", Aerospace Corp (1968).

[13] Teidelt, E., Starcevic, J, and Popov, V., "Influence of ultrasonic oscillation on static and sliding friction," Tribology Letters, 1, 51-62 (2012).

[14] Dong, S. and Dapino, M., "Piezoelectrically-induced ultrasonic lubrication by way of Poisson effect " Proc. SPIE, 8343L (2012).

[15] Dong, S. and Dapino, M., "Wear reduction through piezoelectrically-assisted ultrasonic lubrication, " Smart. Mater. Struct. 23104005 (2014).

[16] Dong, S. and Dapino, M., "Mapping of power consumption and friction reduction in piezoelectrically-assisted ultrasonic lubrication" Proc. SPIE, 943304 (2015).

[17] Dong, S. and Dapino, M., "Elastic-plastic cube model for ultrasonic friction reduction via Poisson effect, " Ultrasonics, 54, 343-350 (2014).

[18] Hunt, F., "Electroacoustics; the analysis of transduction, and its historical background," Harvard University Press, Cambridge, 1954.

[19] Downey, P. and Dapino, M., "Extended frequency bandwidth and electrical impedance tuning in hybrid Terfenol-D/PMN-PT transducers in mechanical series configuration," J. Intell. Mater. Sys. Struct. 15 (2005).

[20] Shi, X., and Polycarpou, A. A., "Measurement and modeling of normal contact stiffness and contact damping at the meso scale," Journal of Tribology, 127, 52-60, 2005. 


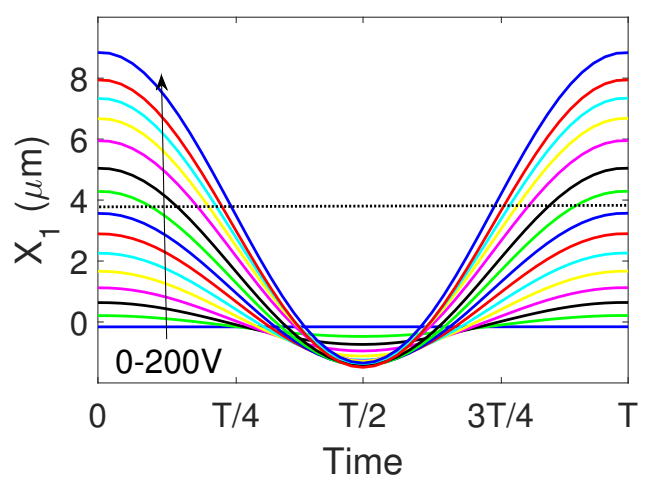

(a)

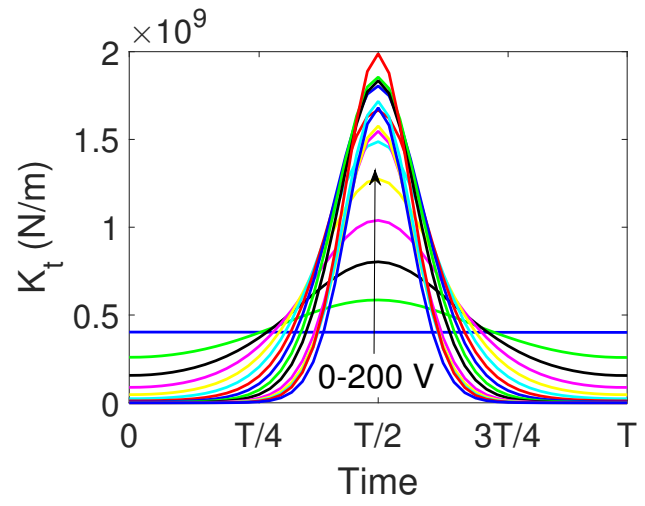

(c)

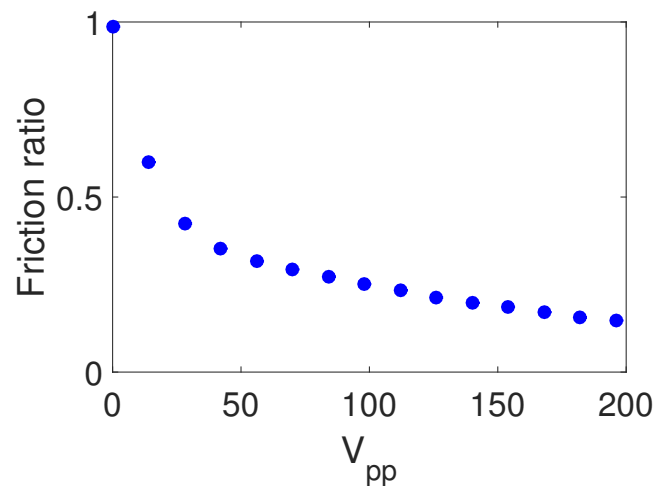

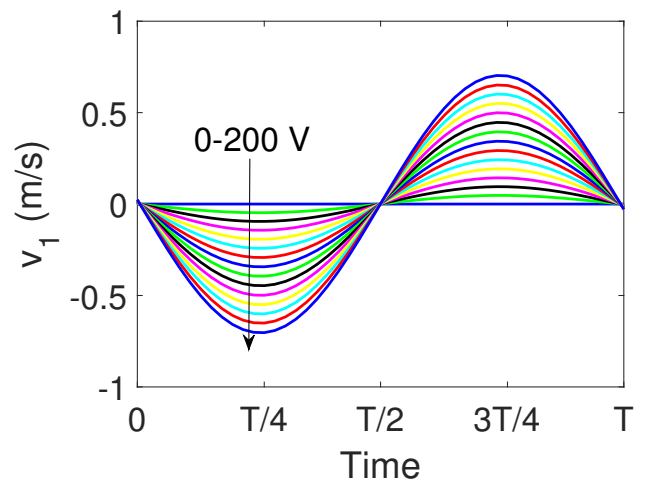

(b)

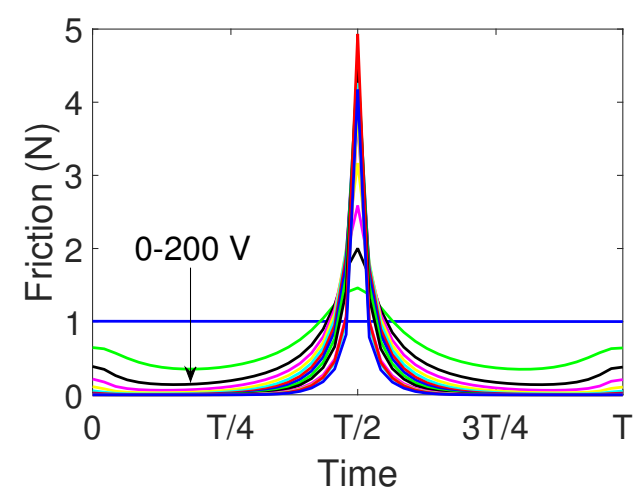

(d)

(e)

Figure 10: Simulation results at various voltages: (a) displacement (dashed line denotes the critical displacement above which the two surfaces separate); (b) velocity; (c) tangential stiffness; (d) instantaneous friction; (e) relationship between voltage and friction ratio. 


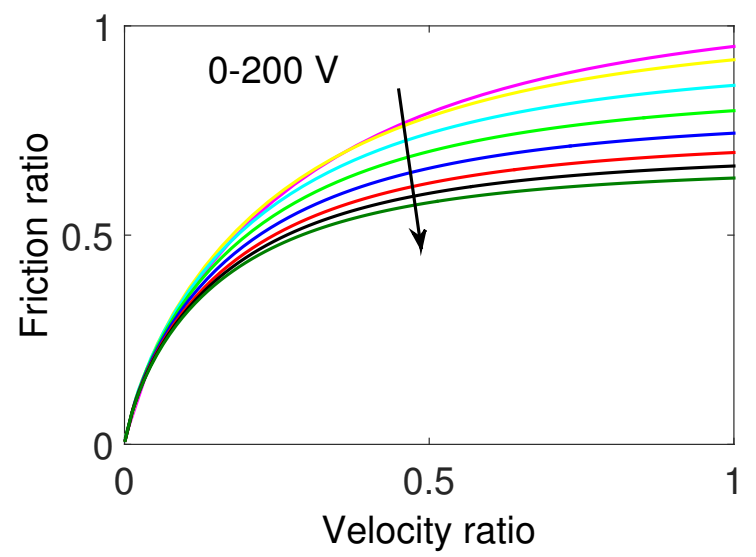

Figure 11: Relationship between friction ratio and velocity ratio.

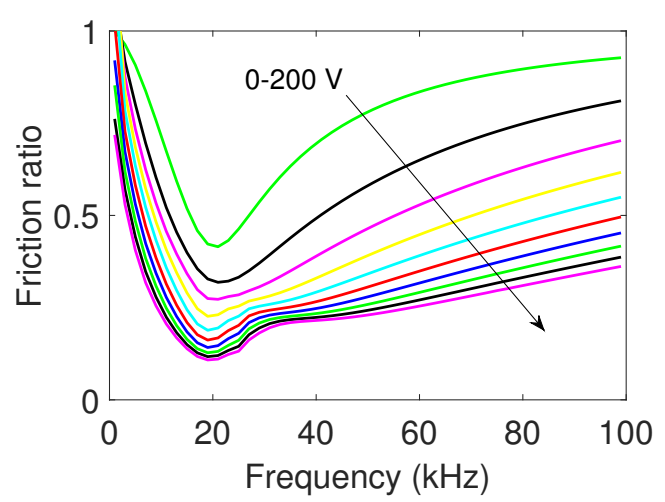

(a)

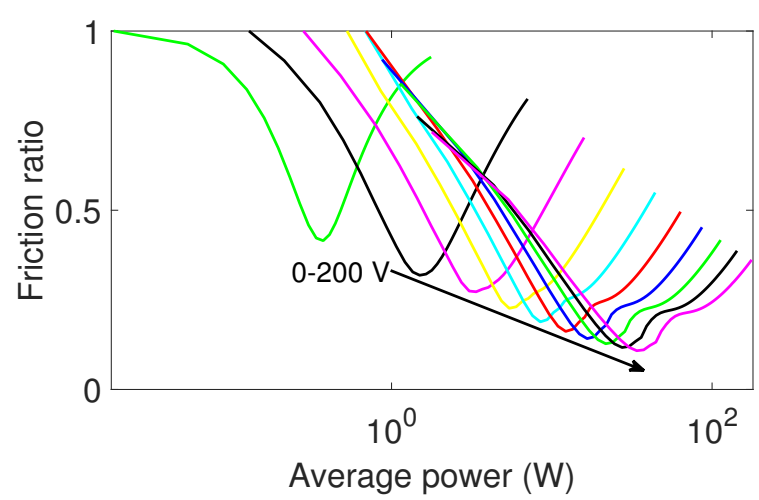

(b)

Figure 12: (a) Relationship between friction ratio and driving frequency; (b) relationship between friction ratio and power consumption. 


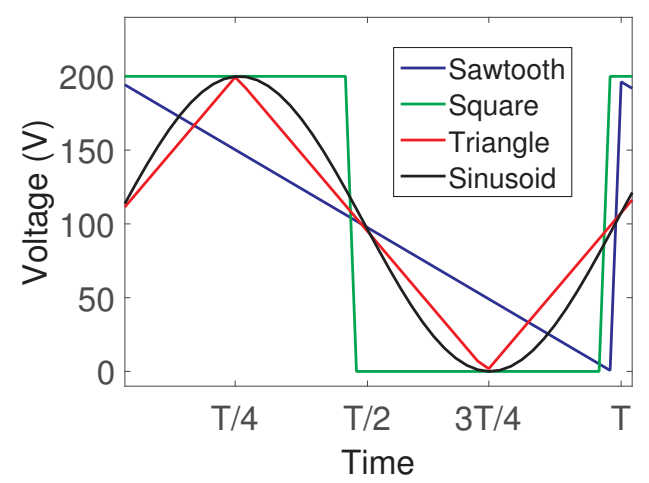

(a)

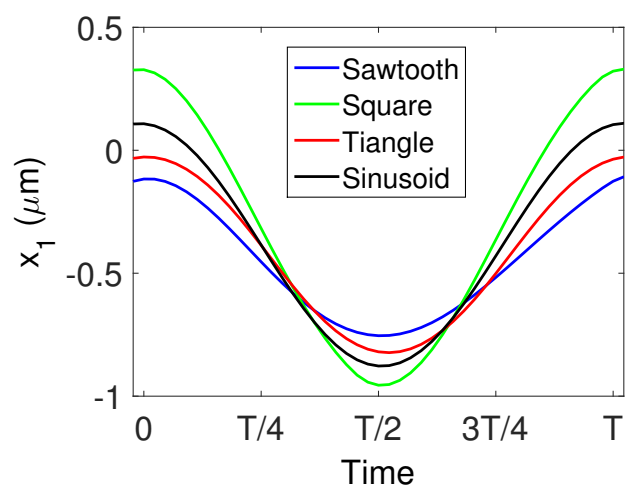

(b)

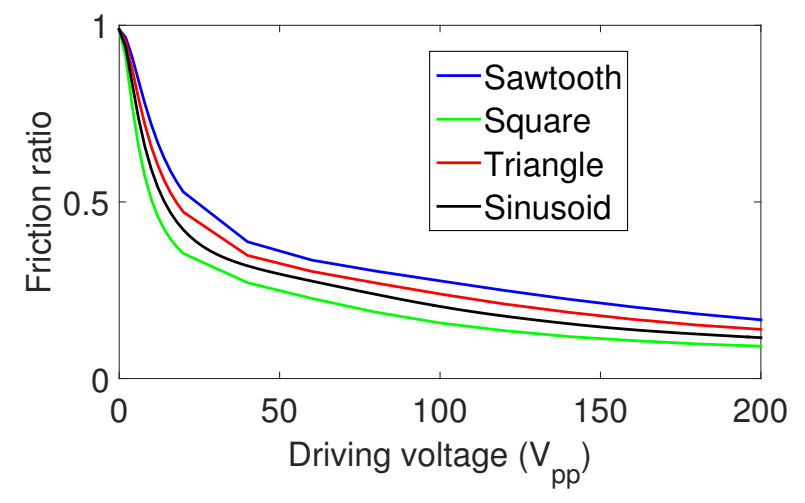

(c)

Figure 13: Parametric study of waveform: (a) waveforms; (b) steady-state displacement at $x_{1}$; (c) relationship between friction ratio and waveform. 